\title{
The Effect of Viagra on Autonomic Function Using Spectral Analysis and Approximate Entropy Method
}

\section{Ahmed Kamal*}

Tennessee Tech University, College of Engineering, MIT Department, PO Box 5003, Cookeville, TN 38505 USA

\begin{abstract}
Objective: Autonomic nervous system involvement in patients with Erectile Dysfunction (ED) has shown conflicting results. Our main purpose was to assess the effect of treatment with Viagra on autonomic function of group of ED patients in comparison with another group with control subjects with ED treated by herbal substances by measuring the frequency gain response of the two groups. Other aim of this study was to determine wither the duration of Viagra treatment correlated with measures of heart rate variability signal (HRV) using approximate entropy index (ApEn).

Methods: Twenty two patients treated for ED by Viagra $(100 \mathrm{mg})$ and twenty one matched control subjects treated ED by herbal were evaluated at Erectile Dysfunction Center, Johns Hopkins Hospital, Maryland, USA during 2012/ 2013 by measuring the breathing at different rates and HRV signal derived from Electrocardiogram (ECG). The autopower and cross power spectra between HRV signal and breathing signal at different breathing rate were calculated and frequency gain response for both groups were obtained. ApEn described as measure of regularity of HRV was calculated for both patients with Viagra treatment and herbal treatment for control subjects.

Results: Both frequencies gain response and ApEn measures were increased in patients treated with Viagra in comparison with control group. The power spectra of patients with Viagra men showed both increase High Frequency (HF) value and higher low frequency bands with respect to control subjects. On other hand, ApEn measure was significantly increased in longer Viagra taking duration than in herbal group.

Conclusion: These findings suggest that the spectral analysis of HRV signal using breathing at different frequencies may detect balance of autonomic of autonomic system of ED patients treated with Viagra specially with increasing the sympathetic activity (higher low frequency) and as well as the parasympathetic tone(higher frequency gain response) which can lead to reduce sudden deaths in patients with treating with viagra. APEn may be marker related to duration of taking Viagra. Further studies are needed to include more patients to strengthen the quantitive and qualitative indices proposed in this paper.
\end{abstract}

Keywords: Heart rate variability; Gain frequency response; Approximate entropy

\section{Introduction}

Erectile dysfunction may produce dysfunction in the autonomic nervous system. However, this relationship has not been fully studied in quantitative way and some studies has conflicting results $[1,2]$. Several studies found more excess cardiovascular mortality in Erectile Dysfunction(ED) patients than in control [3-6], and cardiac failure and sudden death $[7,8]$. Recent attention has focused on the importance of long term pattern in heart rate i.e. Heart Rate Variability (HRV) to assess and quantify the autonomic function using simple statistical measures and power spectral analysis.

The aim of this study to investigate the auto power spectra of HRV signals at different breathing rates as well as producing the gain frequency response for both ED subjects group (Control) using herbal treatment (Not Viagra) and ED patients group treated with Viagra . Another aim of this study is to introduce other statistics to assess the autonomic function developed from nonlinear dynamics. One such statistics, Approximate entropy (ApEn) which quantifies the regularity of patterns in data set [9-14].ApEn has been applied to HRV signal for both control and ED patients with Viagra.

\section{Material and Methods}

The study group was composed of twenty one control subjects (No Viagra treatment) $(53 \pm 5.7)$ years and thirty two patients with Viagra treatment $(55 \pm 4.6)$ years has been investigated in this study at Johns Hopkins hospital, MD, USA during 2011/ 2012. None of ED patients in both two groups had clinical signs of myocardial infarction, arterial hypertension, or pulmonary disease. No drugs that could affect HRV parameters were used by the subjects and the control groups under study. Four ED patients who were smoking at least one cigarette per day were excluded from this study. Therefore the final group consisted of $22 \mathrm{ED}$ patients treated with Viagra. All subjects agreed to participate in the research with signing a consent letter prior to their inclusion in the study and the consent of ethical committee was obtained and approved the study protocol. Each subject lies supine on a bed. The breathing signal is measured using a thermistor placed in the nose. The Electrocardiogram (ECG) is taken from wrists and ankle (Lead II). All the measurements were recorded for the duration of the experiments using laptop computer. After the subject has settled comfortably, a base line of the physiological measurements, ECG, derived HRV and breathing signal were measured for $5 \mathrm{~min}$ for both healthy subjects and patients with diabetes at rest in supine position to calculate the ApEn and heart rate for each group. The subject is then asked to breathe deeply at different rates for 2 min following the light sequence, green inhale and red exhale. The cycle length is varied using frequency generator and the light indicator automatically divides the cycle length

*Corresponding author: Ahmed Kamal, MET Department, College of Engineering Tennessee Tech University Box 5003,Lewis Hall, Cookeville, TN 38501,USA, Tel: 931 239-7570; E-mail: AKamal@tntech.edu

Received February 23, 2015; Accepted March 19, 2015; Published March 25, 2015

Citation: Kamal A (2015) The Effect of Viagra on Autonomic Function Using Spectral Analysis and Approximate Entropy Method. Int J Neurorehabilitation 2: 156. doi: $10.4172 / 2376-0281.1000156$

Copyright: () 2015 Kamal A. This is an open-access article distributed under the terms of the Creative Commons Attribution License, which permits unrestricted use, distribution, and reproduction in any medium, provided the original author and source are credited. 
into $40 \%$ inhalation and $60 \%$ exhalation [15]. A rest period is allowed between each breathing rate sequence. The following breathing rates were examined for each subject $3,4,6,10,12,14,16,18,24$ and 30 breath/ min. Figure 1 shows the block diagram of the experiments.

\section{Measurement and analysis of physiological signals}

The ECG for every patient participated in this study is fed into an electronic device which detects the R-wave and measures the time until the next R wave occurs. Alternately, the peak of QRS was identified for each beat using rate-detector algorithm after exclusion of artifacts and ectopics. Those periods in which beat identification was poor were excluded from the analysis. This R-R time is converted into voltage directly proportional to that time. The reconstructed voltage signals as shown in figure 2 may now placed as HRV signals and can be interfaced to Laptop computer to obtain autopower and cross power spectra of HRV signal and breathing signals at different rates using Fast Fourier transform(FFT) to calculate the gain frequency response and ApEn (Appendix)

\section{Identification procedure of frequency gain response}

Consider a linear system with an input-output relationship as shown in Figure 3. It can be represented by a transfer function which can be found by several conventional methods e.g. impulse response, $\mathrm{H}(\mathrm{jw}) \cdot \mathrm{R}(\mathrm{jw})$, where $\mathrm{H}(\mathrm{jw})$ and $\mathrm{R}(\mathrm{jw})$ are the Fourier transform (using FFT algorithm) of the output signal $\mathrm{h}(\mathrm{t})$ and input signal $\mathrm{r}(\mathrm{t})$, respectively. If we define the transfer function between $\mathrm{H}(\mathrm{jw})$ and $\mathrm{R}(\mathrm{jw})$ as shown in Figure 3 as

$$
\mathrm{Grh}=\frac{\mathrm{H}}{\mathrm{R}}(1)
$$

And multiply the numerator and denominator of the right side by $R^{\star}(j w)$,i.e. the complex conjugate of the Fourier transformed input $r(t)$, the result is

$$
\mathrm{Grh}(\mathrm{jw})=\frac{\mathrm{HR}^{*}}{\mathrm{RR} *}=\frac{\operatorname{Prh} *(\mathrm{jw})}{\operatorname{Prr}(\mathrm{w})}
$$

Where $\operatorname{Prh}(\mathrm{jw})$ is the complex conjugate of the cross power spectrum between $r(t)$ and $h(t)$ and $\operatorname{Prr}(w)$ is the auto-power spectrum of $r(t)$.Assuming that $n(t)$ and $r(t)$ are not auto-correlated then $\operatorname{Prh}^{*}(j w)$ is not influenced by the presence of $n(t)$. Since $\operatorname{Pr}(w)$ does not involve

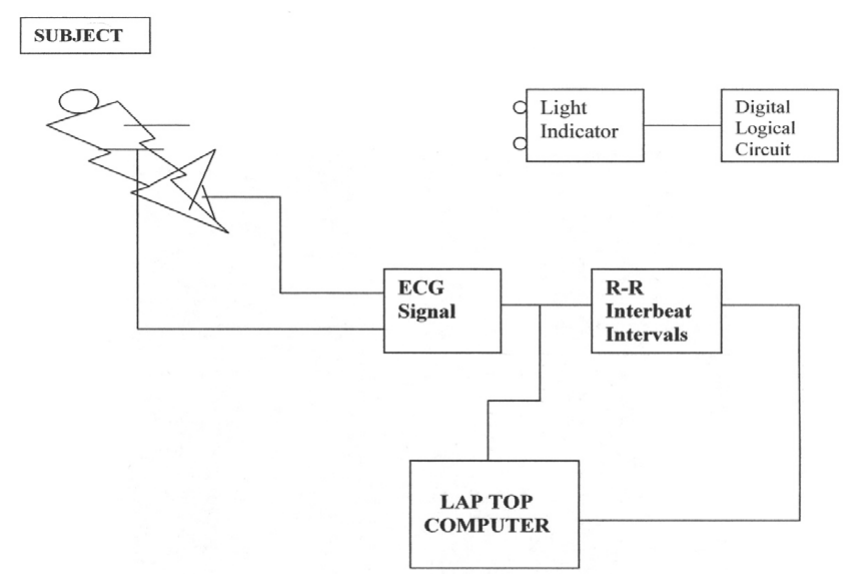

Figure 1: Derivation of heart rate variability (HRV) signal from Electrocardiogram (ECG). (a) ECG (b) Detection of R-R interval(c) Construction of HRV signal (d) Smoothed HRV signal

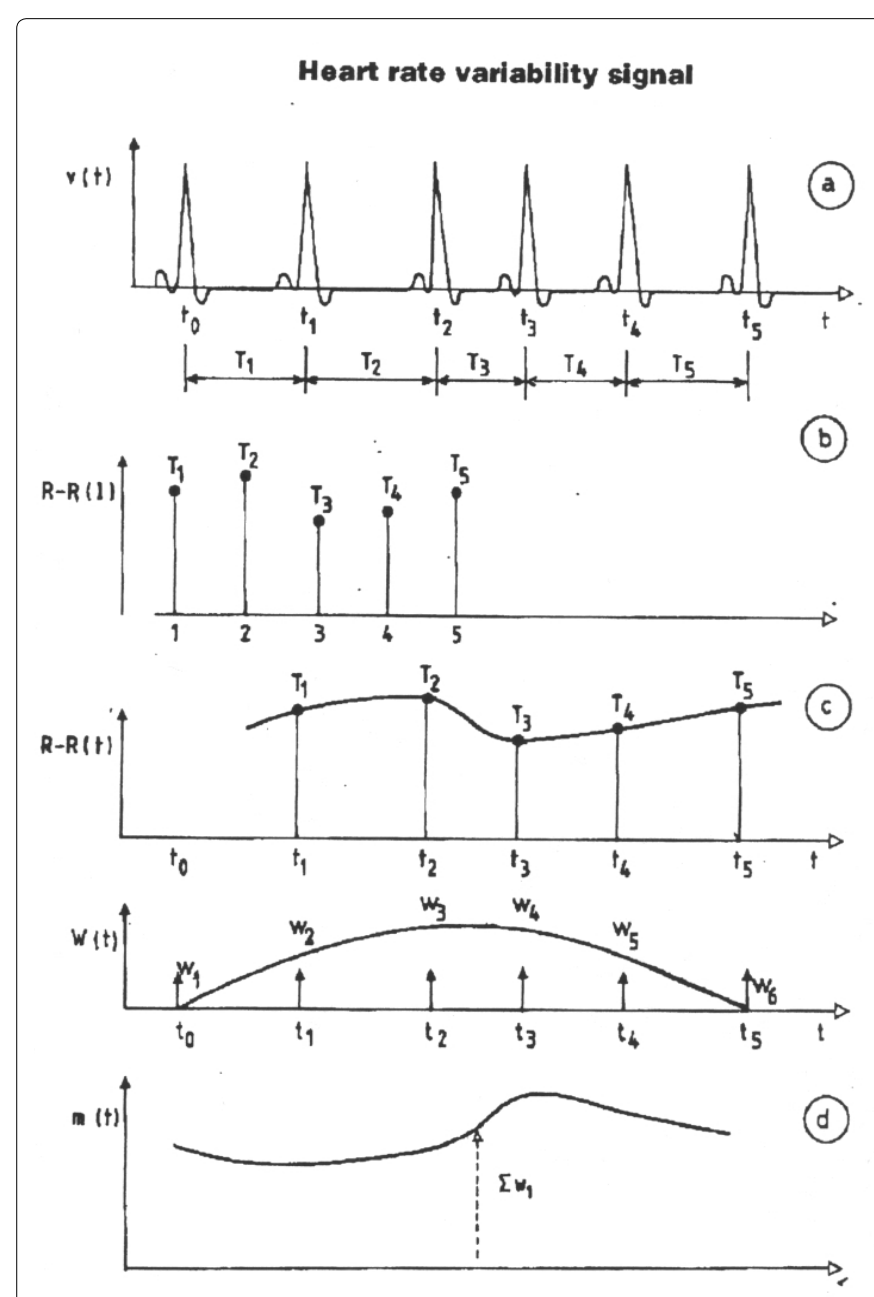

Figure 2: Block diagram of experimental procedure

\begin{tabular}{|l|c|c|}
\hline Variable & Control $(\mathrm{n}=21)$ & EDPatients $(\mathrm{n}=22)$ \\
\hline Age (years), mean $\square \square$ SD & $52 \pm 1.4$ & $55 \square 1.6$ \\
\hline $\mathrm{BMI}(\mathrm{kg} / \mathrm{m} 2)$ & $22.1 \pm 2.1$ & $25.2 \square \square 4.6$ \\
\hline Mean disease duration (years) & 2.3 & 7.2 \\
\hline ESR (mean $\square$ SD) & $38.7 \pm 16.4$ & $41.6 \square \square 25.1$ \\
\hline CRP (mean $\square$ SD) & $39.6 \pm 86.4$ & $51.4 \square \square 124.6$ \\
\hline $51.4 \square 124.6)$ & & \\
\hline
\end{tabular}

Table 1: Clinical characteristics of patients ( BMI ,body mass index, CRPC-reactive protein, ESR, erythrocyte sedimentation rate)

$\mathrm{n}(\mathrm{t})$ at all, this estimate of $\mathrm{Grh}(\mathrm{jw})$ from $\mathrm{h}(\mathrm{t})$ and $\mathrm{r}(\mathrm{t})$ becomes a good method of overcoming the presence of noise to estimate $H / R$ or the frequency gain response.

\section{Results}

The clinical data of twenty two patients of ED are summarized in Table 1. There were no significant differences between two groups in these parameters, only in CRP C-reactive protein which has high level in ED patients treated by Viagra than in control. All ED patients were under clinical supervision with adjusted blood sugar control.

However, the results of HRV analysis and Approximate Entropy (ApEn) are shown in Figure 4 and Table 2 and Table 3. Figure 4 shows the gain frequency response for both ED Patient group and control 


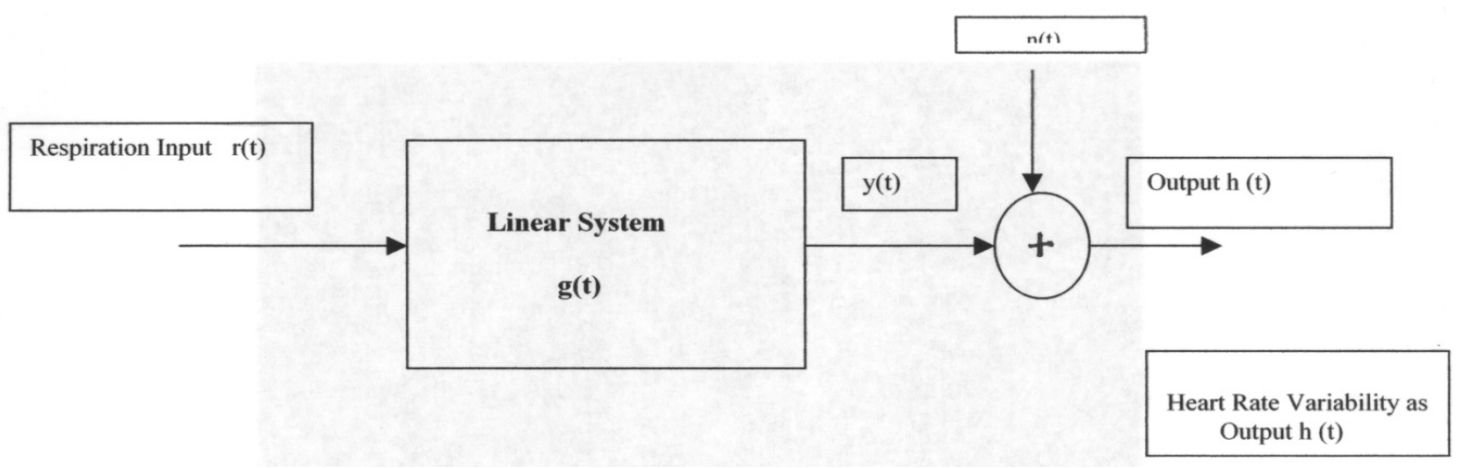

Figure 3: Relationship between input(breathing) and output(HRV signal).

\begin{tabular}{|l|c|c|c|}
\hline & $\begin{array}{c}\text { Group }(\mathbf{n}=22) \\
\text { Viagra taking }\end{array}$ & $\begin{array}{c}\text { Control Group }(\mathbf{n}=21) \\
\text { No Viagra Taking }\end{array}$ & P value \\
\hline ApEn & $0.86 \pm 0.15$ & $0.78 \pm 0.1$ & $<0.05$ \\
\hline $\begin{array}{l}\text { Heart rate } \\
\text { beats/sec) }\end{array}$ & 70.6 & 75.4 & 0.15 \\
\hline SDNN(ms) & $132.6 \pm 36.8$ & $88.3 \pm 18.4$ & 0.003 \\
\hline
\end{tabular}

Table 2: ApEn and HRV parameters (heart rate and SDNN(standard deviation of all $\mathrm{R}-\mathrm{R}$ intervals) at rest in patients with Viagra and control.

\begin{tabular}{|l|c|c|c|}
\hline $\begin{array}{l}\text { Duration Of } \\
\text { ED with Viagra }\end{array}$ & $3-6$ years $(n=11)$ & $1-3$ years $(n=21)$ & P value \\
\hline ApEn & $0.86 \pm 0.09$ & $0.75 \pm 0.1$ & 0.001 \\
\hline $\begin{array}{l}\text { Heart rate } \\
\text { (beats/sec) }\end{array}$ & $70 \pm 2.6$ & $76 \pm 3.2$ & NS \\
\hline SDNN(ms) & $102 \pm 8.6$ & $94 \pm 10.3$ & NS \\
\hline
\end{tabular}

Table 3: HRV parameters( heart rate and SDNN( standard deviation of all R-R intervals) and ApEn at rest in patient with ED according to duration of ED disease.

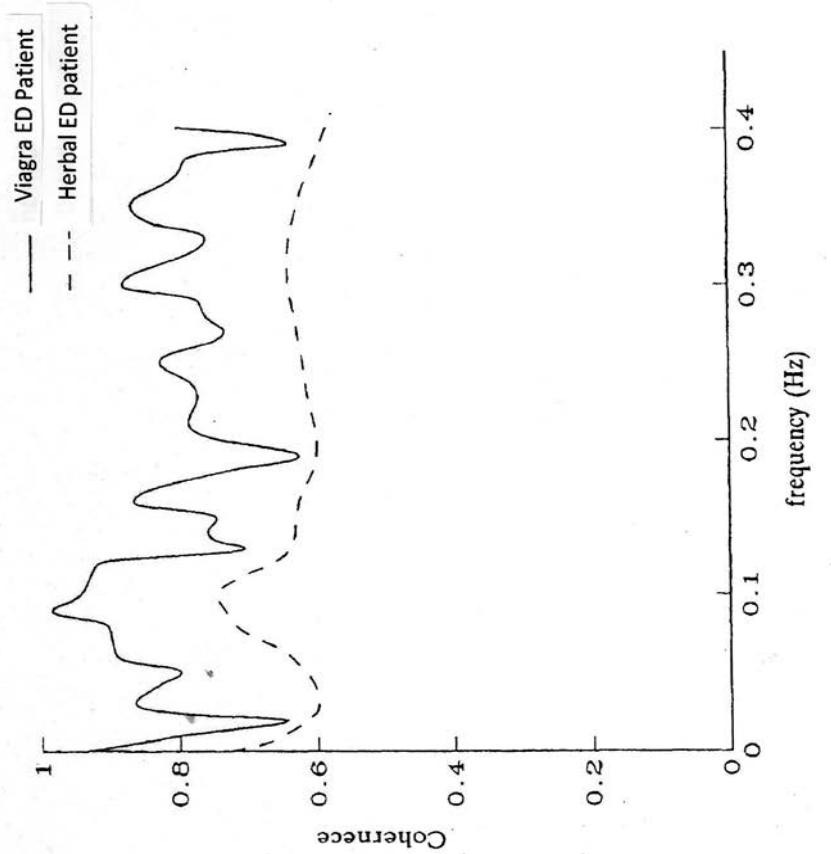

Figure 4: The average frequency gain response of Typical control ED subjects treated by herbal substances and Typical ED patients treated by Viagra at different breathing rates. group at different breathing rates. The gain frequency response of ED Patients group treated by Viagra is significantly higher $(\mathrm{p}=0.003)$ than ED Control group treated by herbal tea. Table 2 shows significant difference $(\mathrm{p}=0.001)$ between the value of ApEn of HRV signal at rest for both groups. Table 3 shows the duration of ED in patients groups and ApEn as well as HRV parameters (heart rate, SDNN (standard deviation of all R-R intervals) at rest.

\section{Discussion}

In this study, we introduced new index ApEn to quantify the regularity of HRV. Lower ApEn indicates lower regularity and less variation of $\mathrm{HRV}$ signals and higher ApEn indicates greater regularity and more variation of HRV [9-14]. Appendix (A) explains the approximate Entropy method. Referring to Table 2, there is significant increase $(p<0.5)$ in both ApEn and SDNN in ED Viagra patients group. Also, Table 3 shows significant increase in ApEn index of ED patients with Viagra treatment related to the duration of the disease. However, SDNN and heart rate did not show significant decrease with the duration of treatment of ED disease by Viagra. This is important finding to quantify the autonomic function of ED patients Viagra treated using ApEn index. In fact, a low ApEn correlates with greater system isolation i.e. less interaction between multiple inputs that makeup a normal control system $[15,16]$

Several normal physiologic processes have been studied to demonstrate the relationship between ApEn and the integrity of the biological system. Kaplan et al. [13] compared healthy young and elderly subjects and found reduced complexity in the elderly. Similarly, Rayan et al. [14] demonstrated ApEn decreased with aging. In this study, we observed the highest normalized ApEn values in ED patients treated with Viagra with improving autonomic dysfunction. Also, we found correlation between increasing ApEn values and Viagra treatment duration as shown in Table 3. The significance of ApEn index is to represent one value (between 0 and 1) which indicates the quality of connection, interaction, regularity and complexity in the system(Appendix A) . Applying this concept to the autonomic nervous system of ED patients treated with Viagra, it seems their autonomic system improved the integrity and interaction with respect to control subjects treated by herbal substances which may be manifested in high ApEn values in ED patients group treated by Viagra as shown in Table 3. The frequency gain response as shown in Figure 4 demonstrates the influence of breathing on gain response with respect to ED patients with Viagra treatment and control subjects. The ED patients group treated by Viagra exhibits two distinct peaks at nearly 6 cycles/min $(0.1 \mathrm{~Hz})$ and nearly $15 \mathrm{cycles} / \mathrm{min}(2.5 \mathrm{~Hz})$.However, control group 
shows only one lower distinct peak at the same breathing rate 6 cycles/ min. It is clear that ED patients treated by Viagra show higher gain response to breathing than the other ED control subjects treated by herbal at all breathing frequencies. This may be attributed to increase in autonomic function in ED group treated by Viagra in both sympathetic and parasympathetic activities in ED patients with Viagra treatment. The ability of Viagra to widen the arteries and lower the blood pressure might be important with respect to the possible prevention of increased rate of sudden death occurred in ED Patients treated with Viagra which correlate with increased parasympathetic activity [7]. The increased activity of sympathetic activity in Viagra group suggests increased thermoregulatory improvement which can help patients with Raynaud's disease [17]. Our finding which correlates the duration of ED disease in Patients group treated with Viagra and ApEn may be considered a simple prognostic value to assess effective improvement of the autonomic function of ED patients treated with Viagra as shown in Table 3.

\section{Conclusion and Future Work}

This study pointed to the following

1- The spectral analysis Method and APEN index are useful to show the effect of Viagra with respect to herbal substance in assessment of Autonomic Function for both groups

2- ApEN index is helpful to assess autonomic function based on the duration of treatment of Viagra to improve the autonomic function.

\section{References}

1. American Heart Association (2002) "Viagra Helps Men With Heart Failure." Science Daily.
2. Bordo S (1998) Pills and power tools. Men and masculinities. 1: 87-90.

3. Boyer, Rodney (2007)Perhaps Viagra Can Soften Jet Lag's Impact. ABC News Medical Unit. In:Interactive Concepts in Biochemistry, John Wiley \& Sons Publishers, Inc.

4. Devettere, Raymond (2002)Introduction to virtue ethics: insights of the ancient Greeks. Georgetown University Press.

5. Diamond, Jed (1998)Male Menopause. Illinois: Sourcebooks.

6. Dobson R (2007) Viagra: Not Just for Erections. The Independent UK.

7. Elsbach, Kimberly (2006)Organizational perception management. Routledge.

8. Michael E (2004) Metz. Coping With ErectileDysfunction: How to Regain Confidence and Enjoy Great Sex, New Harbinger Publications, Boston, USA.

9. Morse SA (2006)The Erectile Dysfunction Solution Guide. Wiley, New York.

10. Pincus S M (1991) Approximate entropy as a measure of system complexity. Proc Natl Acad Sci USA 88:2297-2301.

11. Pincus SM (1994) Greater signal regularity may indicate increased system isolation. MathBiosc,122: 161-181

12. Pincus SM, Goldberg AL (1994) Physiological time series analysis: What does regularity quantify? Am J Physiol 266: H 1643-1656.

13. Kaplan DT, Furman MI, Pincus SM ,Rayan SM, Lipsitz LA, et al (1991) Aging and the complexity of cardiovascular dynamics. Biophys J 59: 945-949.

14. Rayan SM, Goldberg AL, Pincus SM, Mietus J, Lipsitz LA (1994) Gender and age related differences in heart rate dynamics: are women more complex than men ? J Am Coll Cardiol 24 : 1700-1707.

15. Friedman D M (2001)A mind of its own.In: Viagra's Other Uses, New York: Penguin Books.

16. Hasty, Susan (2005) Viagra used in stroke recovery study. Physician Business Week.

17. Finn, Robert (2005)Vacuum constriction can benefit erectile dysfunction International Medical News Group, New York. 